\title{
MONITORAMENTO DO PROCESSO DE CORTE A LASER DE CERÂMICAS AVANÇADAS USANDO DIAFRAGMAS PIEZELÉTRICOS DE BAIXO CUSTO
}

Ana Carolina Balarin de Andrade*1; Martin Antonio Aulestia Viera2; Paulo Roberto de Aguiar2; Fabio Romano Lofrano Dotto2; Felipe Aparecido Alexandre2; Eduardo Carlos Bianchi $^{1}$; Thiago Valle França ${ }^{1}$

${ }^{1}$ Departamento de Engenharia Mecânica, Universidade Estadual Paulista Júlio de Mesquita Filho - UNESP, Av. Eng. Luiz Edmundo Carrijo Coube,14-01; Bauru/SP; *ac.andrade@unesp.br

2 Departamento de Engenharia Elétrica, Universidade Estadual Paulista Júlio de Mesquita Filho - UNESP, Av. Eng. Luiz Edmundo Carrijo Coube,14-01; Bauru/SP

Resumo: As cerâmicas avançadas são muito utilizadas em diversas aplicações industriais devido às suas propriedades. No entanto, a usinagem de componentes cerâmicos é muito difícil por causa da sua alta dureza e fragilidade. O presente trabalho apresenta a utilização de um diafragma piezelétrico de baixo custo no monitoramento da largura do dano no processo de usinagem a laser de cerâmicas avançadas. Os ensaios foram realizados para diferentes condições de corte, sendo os sinais coletados por um sistema de aquisição de dados. O valor médio quadrático foi obtido a partir do sinal filtrado em uma determinada banda de frequência previamente selecionada. Os resultados demonstraram que o transdutor pode ser usado eficazmente para o monitoramento do processo de corte a laser.

Palavras-Chave: corte a laser; cerâmicas avançadas; PZT; processamento de sinais.

\section{MONITORING OF CERAMIC LASER CUTTING USING LOW-COST PIEZOELECTRIC DIAPHRAGM}

\begin{abstract}
Advanced ceramics are widely used in industrial applications due to their properties. However, machining of ceramic components is very difficult because of their high hardness and brittleness. The objective of the present work is to monitor the damage width in the ceramic laser machining process through low-cost piezoelectric diaphragms. Tests were performed for different cutting conditions, and the signals were collected by a data acquisition system. The root mean square value was obtained from the filtered signal in a previously selected frequency band. Results indicate that the transducer can be used effectively to monitor the laser cutting process.
\end{abstract}

Keywords: laser cutting; advanced ceramics; PZT; signal processing. 


\section{INTRODUÇÃO}

As cerâmicas avançadas têm sido frequentemente utilizadas na indústria por causa das suas propriedades, como estabilidade química a altas temperaturas, resistência, dureza, baixo peso específico, entre outros. No entanto, as principais características das cerâmicas podem ser uma barreira limitadora em sua usinagem [1].

Como há uma dificuldade para usinar cerâmicas utilizando métodos tradicionais, a usinagem a laser surgiu como uma técnica em potencial. Por se tratar de um processo térmico, as propriedades térmicas das cerâmicas, como baixa condutividade e difusividade térmica, as tornam adequadas para usinagem. Lasers são amplamente utilizados na indústria devido à precisão de operação, baixo custo, processamento localizado e alta velocidade de operação. Sendo assim, a usinagem a laser tem várias vantagens sobre os processos convencionais, como também a falta de desgaste e desvios da ferramenta de corte, bem como nenhum efeito de vibração [1-3].

O mecanismo de remoção de material durante a usinagem a laser consiste na focalização do feixe do laser na superfície da peça, desta forma a energia térmica é absorvida pelo material, levando ao seu aquecimento até a sua fusão, vaporização ou mudança de estado químico. Um fluxo de gás é utilizado para garantir que esse material seja removido. O aquecimento é feito de forma localizada, e pode ser controlado por meio das configurações ópticas do feixe de laser [2-5].

Uma nova tecnologia de usinagem híbrida foi recentemente desenvolvida por Guerrini et al. [6], capaz de induzir trincas em um volume definido de material através de um tratamento a laser. O processo resume-se em usinar com o laser (induzindo as trincas) antes de outro processo de usinagem, como exemplo a retificação. A geração de trincas controladas facilita a remoção de material, o que a torna muito interessante para usinagem de cerâmicas avançadas. Uma forma de garantir o controle do processo a laser é através do seu monitoramento.

O monitoramento de usinagem aplicado a materiais duros ou quebradiços, como as cerâmicas, proporciona informações valiosas sobre o processo de fabricação. Os sensores de emissão acústica (EA) detectam ondas acústicas emitidas pelo material ao ser exposto a uma tensão ou deformação, e o seu uso para monitorar processos de usinagem é de grande eficácia. Segundo Lauro et al. [7] a EA é considerada um dos métodos de monitoramento de usinagem mais precisos para a identificação de danos.

Viera et al. [8] propuseram a aplicação de transdutores piezelétricos de baixo custo na análise da qualidade superficial de componentes cerâmicos durante o processo de retificação. Os resultados obtidos mostram uma grande similaridade entre a resposta do sensor PZT de baixo custo e um sensor convencional de EA. Devido ao seu custo e fácil utilização, os transdutores piezelétricos de baixo custo podem ser considerados uma boa alternativa como sensores.

Os sinais obtidos pelo sensor PZT trazem informações do processo, e o processamento destes sinais é feito aplicando estatísticas, que tem o objetivo de 
extrair dos sinais as características que se correlacionam com um determinado fenômeno estudado. A estatística RMS (Valor médio quadrático) é a mais utilizada no monitoramento de sinais e pode ser expressa pela equação (1):

$$
\mathrm{EA}_{\mathrm{rms}}=\sqrt{\frac{1}{\mathrm{~N}} \sum_{\mathrm{i}=1}^{\mathrm{N}} \mathrm{EA}^{2}(\mathrm{i})}
$$

Onde EA é o sinal puro de emissão acústica e $\mathrm{N}$ é o número de amostras discretas (i) consideradas no cálculo [9].

Além do cálculo do RMS, outro parâmetro utilizado na análise de sinais é o cálculo da DFT (Discrete Fourier Transform), que transforma o sinal, que está no domínio do tempo, para o domínio da frequência. O cálculo da DFT é usualmente aplicado devido a sua simplicidade, e pode ser obtido de forma eficiente usando a FFT (Fast Fourier Transform). A FFT é uma implementação simplificada e altamente otimizada da DFT, ambas produzem resultados idênticos [10]. A FFT pode, de forma precisa, separar as componentes harmônicas do sinal coletado.

Sendo assim, o objetivo deste trabalho é o monitoramento da largura do dano no processo de usinagem a laser em cerâmicas avançadas, utilizando transdutores piezelétricos de baixo custo, de forma a relacionar o sinal com as características do corte. A aplicação de diafragmas piezelétricos no monitoramento do processo de corte a laser não foi encontrada na literatura, tornando este trabalho uma abordagem nova e de grande importância para a indústria.

\section{METODOLOGIA}

\subsection{Banco de Ensaios}

Os ensaios foram realizados em uma máquina de modelo CNC Router A12060, da empresa JDR, com um laser acoplado feito de semicondutor com potência máxima de $15 \mathrm{~W}$. Para os ensaios foi utilizada uma peça de alumina ( $96 \%$ de óxido de alumínio e $4 \%$ de outros óxidos) com $110 \mathrm{~mm}$ de comprimento $\times 33 \mathrm{~mm}$ de largura $\times 9 \mathrm{~mm}$ de altura, possuindo microdureza Vickers de $1339 \pm 47$ HV1 (Norma JIS R1610-1991). No total foram realizados seis cortes na peça de alumina, com $30 \mathrm{~mm}$ de comprimento, variando-se a velocidade de corte do laser a cada dois cortes. As velocidades de corte utilizadas foram: $6 \mathrm{~mm} / \mathrm{s}, 12 \mathrm{~mm} / \mathrm{s}$ e $20 \mathrm{~mm} / \mathrm{s}$. Todos os cortes foram realizados utilizando a potência máxima da máquina de corte a laser. Um pincel marcador permanente, da marca Faber Castell na cor preta, foi usado para pintar a superfície da peça de alumina (que possui uma coloração branca) de maneira a evitar reflexão indesejada, garantindo efetiva absorção da energia do laser durante o corte.

Um diafragma PZT de baixo custo, modelo 7BB-20-6, da empresa Murata Eletronics North America, foi utilizado como sensor de atividade acústica nos ensaios. De acordo com De Freitas \& Baptista [11], o diafragma PZT de $20 \mathrm{~mm}$ de diâmetro externo apresenta uma maior sensibilidade, em seu uso como sensor, em relação a outros modelos da marca Murata. Os autores também mostraram que esse sensor possui uma boa resposta operacional até $250 \mathrm{KHz}$, para frequências maiores começa a apresentar atenuações. 
Durante o ensaio, um oscilógrafo, modelo DL850, da empresa Yokogawa, foi empregado para aquisição dos sinais a uma frequência de amostragem de $2 \mathrm{MHz}$. Um amplificador, com um ganho de 25 , também foi utilizado para amplificar o sinal de tensão coletado pelo oscilógrafo.

A Figura (1) mostra uma representação do banco de ensaios utilizado.

Figura 1. Representação dos elementos do banco de ensaios

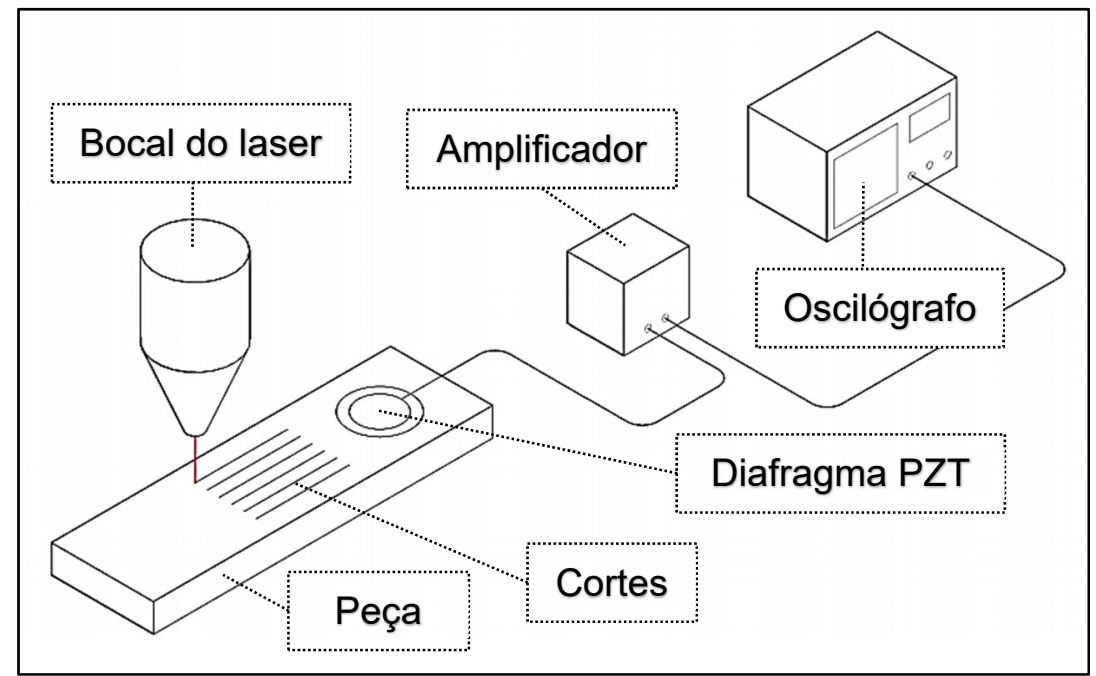

\subsection{Avaliação dos cortes da peça}

A avaliação dos cortes da peça foi realizada por meio de um microscópio digital de $30 \mathrm{~W}$ e 1000x. Foram medidas as larguras dos cortes para as diferentes velocidades de corte. Primeiramente, foi efetuada a limpeza da tinta com uma solução a base de acetona. Após a limpeza, os cortes ficaram visíveis, facilitando a leitura pelo microscópio digital. Para cada corte foram selecionados seis pontos equidistantes, nos quais imagens digitais foram obtidas e, posteriormente, as medidas foram realizadas. Com esses valores, a média e o desvio padrão foram calculados.

\subsection{Processamento digital de sinais}

Os sinais coletados foram digitalmente processados no MATLAB. Os sinais puros do sensor PZT foram primeiramente filtrados na sua frequência operacional que vai até $250 \mathrm{kHz}$.

Em seguida, os sinais foram divididos em janelas de 2048 pontos, representando intervalos de aproximadamente $1 \mathrm{~ms}$, e o valor RMS foi calculado para cada intervalo. Com os valores RMS, calculou-se a média e o desvio padrão para cada condição de corte

Um estudo dos espectros dos sinais foi realizado para identificar bandas de frequência que apresentam uma menor sobreposição e que se correlacionam com as condições de corte da peça, para isso fez-se o uso do cálculo da FFT. Após a seleção 
da banda de frequência, um filtro digital do tipo passa banda foi aplicado nos sinais, e o cálculo do RMS foi refeito para o sinal filtrado.

\section{RESULTADOS E DISCUSSÃO}

Com os resultados das imagens do microscópio digital foi possível observar uma variação na largura do corte para os diferentes tipos de velocidades de corte. A Figura (2) mostra um aumento nos valores das larguras para velocidades de cortes mais baixas. A velocidade de corte está relacionada com o tempo de interação do laser com o material a ser cortado, onde para velocidades de corte mais altas se tem um menor tempo de interação laser / peça, enquanto para velocidades mais baixas se tem um menor tempo de usinagem.

Para a velocidade de $6 \mathrm{~mm} / \mathrm{s}$ foi possível observar uma largura maior do corte. Para tempos de interação menores se tem uma maior exposição do sólido ao laser, gerando uma maior absorção do mesmo e, consequentemente, aumentando o calor gerado. Com isto, como demonstrado nos valores de desvio padrão, observa-se para a velocidades de $6 \mathrm{~mm} / \mathrm{s}$ que a alta temperatura gerada durante o corte resultou a formação de danos maiores no material cortado. Por outro lado, para a velocidade de $20 \mathrm{~mm} / \mathrm{s}$, tem-se um dano menor. Como se trata de uma velocidade consideravelmente maior, o tamanho da largura do corte foi menor devido ao pouco tempo de interação [12,13].

Figura 2. Valores da largura do corte

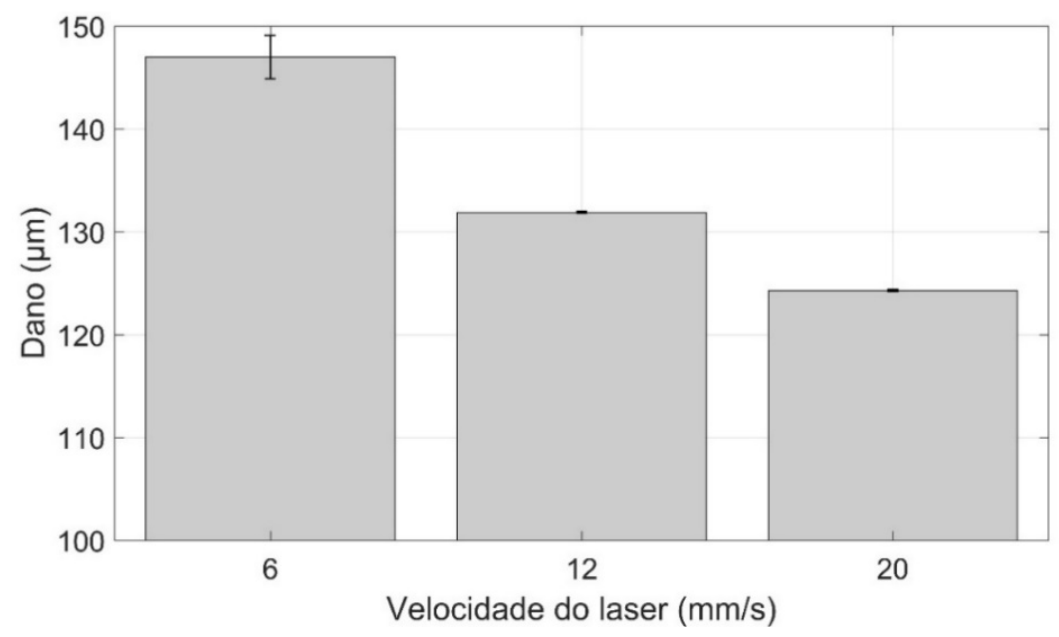

Os valores médios RMS calculados para as velocidades de corte de $6 \mathrm{~mm} / \mathrm{s}$, $12 \mathrm{~mm} / \mathrm{s}$ e $20 \mathrm{~mm} / \mathrm{s}$ são apresentados na Figura (3). Os resultados obtidos não foram satisfatórios, tendo em vista que o comportamento não apresentou uma correlação com as condições de danos do corte.

Quando não filtrados, os sinais apresentam uma grande incidência de ruídos e vibrações do sistema, o que dificulta a detecção da atividade acústica pelo sensor 
diretamente relacionada com as condições dos cortes realizados. Desta forma, analisou-se o espectro do sinal puro do sensor obtido pela aplicação da FFT no MATLAB, sendo selecionada uma banda de frequência que permitiu observar apenas as componentes em frequência de interesse do sinal.

Figura 3. Valores RMS do sinal puro

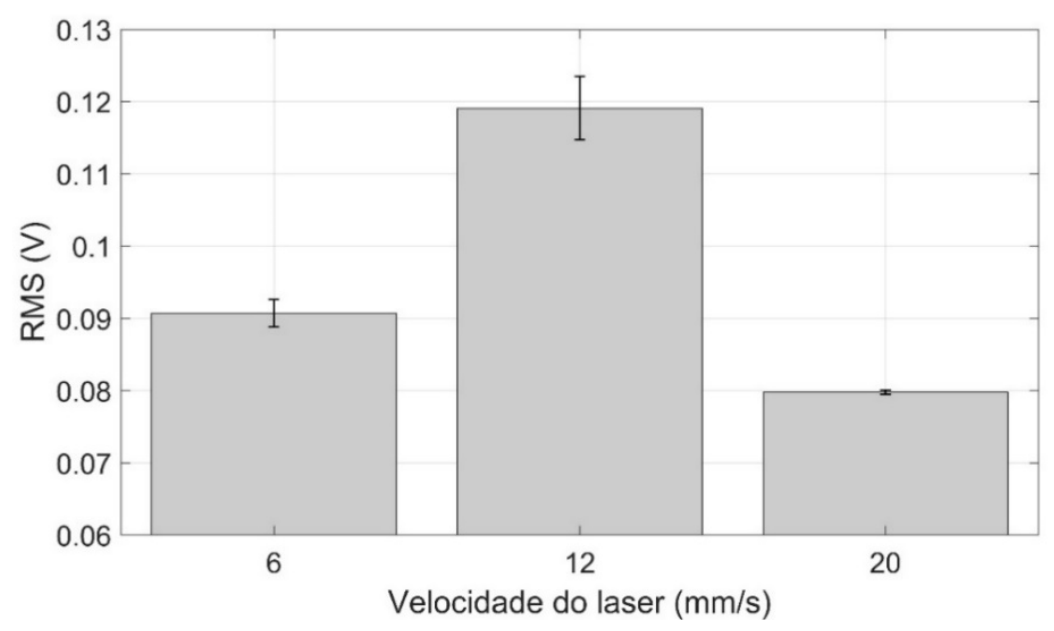

A Figura (4) mostra o espectro da banda de frequência escolhida de $75-80 \mathrm{kHz}$. A seleção da banda de frequência foi feita se levando em consideração janelas de frequência que apresentavam diferenças significativas de magnitude do sinal para cada condição observada [5]. Pode ser observado que as curvas da magnitude do sinal PZT possuem relação com as condições de danos dos cortes. Para a velocidade de corte de $6 \mathrm{~mm} / \mathrm{s}$, o sinal mostra uma atividade acústica maior em relação as outras velocidades, o que é relacionado pela presença de um dano maior. Para o caso da velocidade de corte de $20 \mathrm{~mm} / \mathrm{s}$, devido ao tempo de usinagem ser muito rápido, há um dano menor que é representado por uma atividade acústica menor do sinal.

Figura 4. Espectro da banda de frequência selecionada

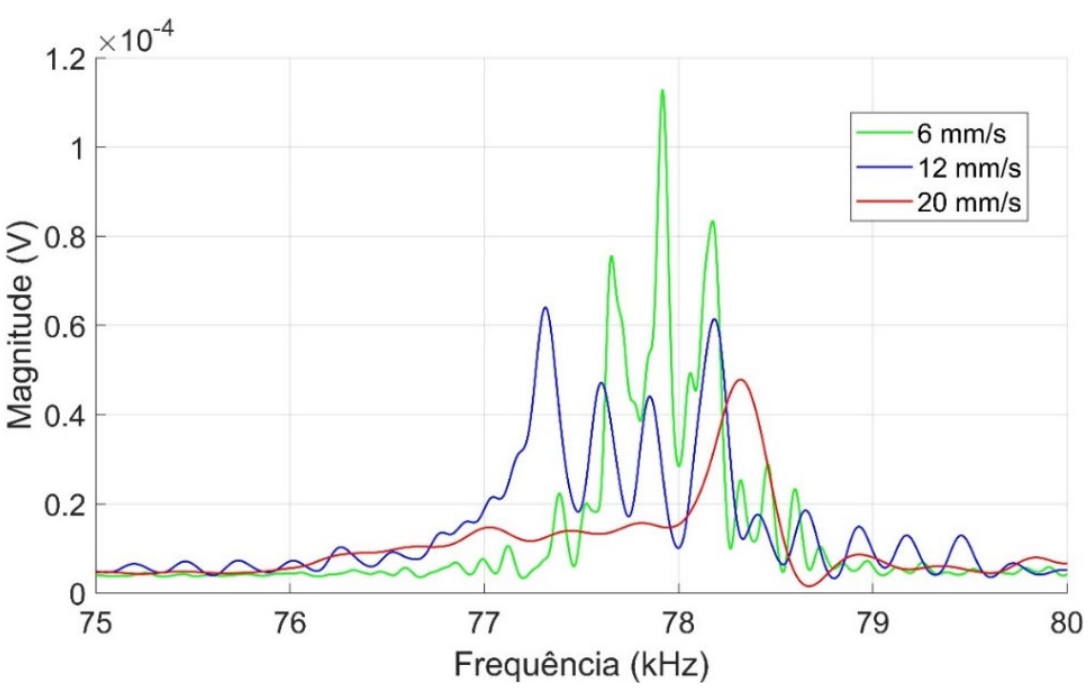


Com a seleção da banda de frequência, o sinal do PZT foi filtrado por um filtro digital passa banda. Os resultados dos valores médios RMS, calculados após o filtro, estão apresentados na Figura (5).

Figura 5. Valores RMS do sinal filtrado

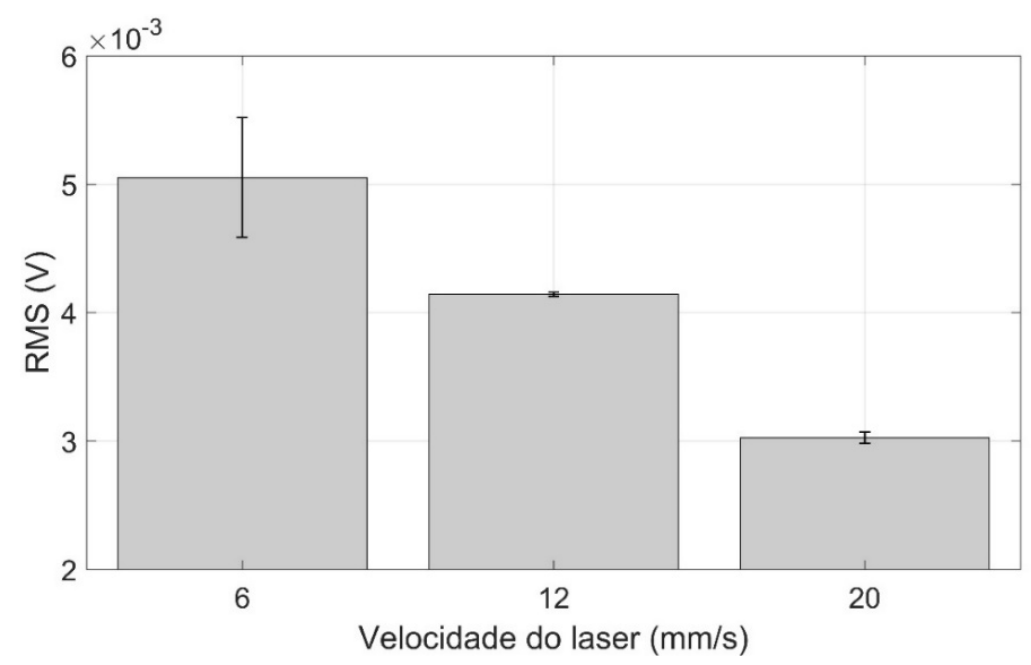

É possível observar na Figura (5) que o comportamento de atividade acústica apresentado na Figura (3) foi corrigido com a aplicação do filtro passa banda. Ao comparar os resultados das Figuras (2) e (5), os comportamentos obtidos e os desvios padrões foram similares, como o esperado. Observa-se que para velocidades mais baixas, há ocorrência de danos maiores e há maior atividade acústica do sinal; esse fenômeno é justificado por uma maior exposição ao calor do laser. Para as velocidades mais altas os danos foram menores, assim como a sua atividade acústica, devido à pouca interação entre o laser e a cerâmica.

\section{CONCLUSÃO}

O propósito deste trabalho é apresentar uma nova técnica de monitoramento de danos durante a usinagem a laser de cerâmicas através de sensores PZT de baixo custo. Diferentes condições de corte a laser em cerâmicas avançadas foram testadas, visando-se auxiliar na identificação dos danos do corte. Os resultados mostram que as condições de corte influenciam de forma direta nos sinais, e mostram uma correlação com os valores da largura do corte. Para velocidades de corte menores, obteve-se resultados de danos maiores e uma maior atividade acústica, e para velocidades de corte maiores os danos são menores e a atividade acústica também é menor. Essa análise foi muito importante para se observar o comportamento do corte a laser para diferentes velocidades, e a sua caracterização pode ser de grande ajuda no controle desse processo de usinagem. 
Agradecimentos

O presente trabalho foi realizado com apoio da Coordenação de Aperfeiçoamento de Pessoal de Nível Superior - Brasil (CAPES) - Código de Financiamento 001, e do Conselho Nacional de Desenvolvimento Científico e Tecnológico (CNPQ) - Código 3006435/2017-9.

\section{REFERÊNCIAS}

1 RAKSHIT, R.; DAS, A. K. A review on cutting of industrial ceramic materials. Precision Engineering, v.59, p. 90-109, 2019.

2 DONG, X.; SHIN, Y. C. "Laser machining and laser-assisted machining of ceramics", in Comprehensive Materials Processing, Elsevier, p. 219-234, 2014.

3 SAMANT, A. N.; DAHOTRE, N. B. Laser machining of structural ceramics-A review. Journal of the European ceramic society, v. 29, n. 6, p. 969-993, 2009.

${ }^{4}$ YILBAS, B. S. "Thermal analysis of the laser cutting process," in The Laser Cutting Process, Elsevier, p. 5-51, 2018.

${ }^{5}$ DUBEY, A. K.; YADAVA, V. Laser beam machining-a review. International Journal of Machine Tools and Manufacture, v. 48, n. 6, p. 609-628, 2008.

6 GUERRINI, G. et al. Hybrid laser assisted machining: a new manufacturing technology for ceramic components. Procedia CIRP, v. 74, p. 761-764, 2018.

7 LAURO, C. H. et al. Monitoring and processing signal applied in machining processes-A review. Measurement, v. 58, p. 73-86, 2014.

8 VIERA, M. A. A. et al. Low-cost piezoelectric transducer for ceramic grinding monitoring. IEEE Sensors Journal, v. 19, n. 17, p. 7605-7612, 2019.

${ }^{9}$ AGUIAR, P. R. et al. In-process grinding monitoring by acoustic emission. In: 2004 IEEE International Conference on Acoustics, Speech, and Signal Processing. IEEE, p. V-405, 2004.

10 ORALLO, C. M. et al. Harmonics measurement with a modulated sliding discrete Fourier transform algorithm. IEEE Transactions on Instrumentation and Measurement, v. 63, n. 4, p. 781-793, 2013.

${ }^{11}$ DE FREITAS, E. S.; BAPTISTA, F. G.. Experimental analysis of the feasibility of lowcost piezoelectric diaphragms in impedance-based SHM applications. Sensors and Actuators A: Physical, v. 238, p. 220-228, 2016.

12 WEE, L. M.; CROUSE, P. L.; LI, L. A statistical analysis of striation formation during laser cutting of ceramics. The International Journal of Advanced Manufacturing Technology, v. 36, n. 7-8, p. 699-706, 2008

${ }^{13}$ BLACK, I. Laser cutting speeds for ceramic tile: a theoretical-empirical comparison. Optics \& Laser Technology, v. 30, n. 2, p. 95-101, 1998. 